\title{
Variations
}

Variations

Revue internationale de théorie critique

$17 \mid 2012$

Critique du travail

\section{Remarques éparses (en style marxiste occidental) sur l'art contemporain, ses problèmes et ses contradictions}

\section{Mikkel Bolt Rasmussen}

Traducteur : José Chatroussat

\section{OpenEdition}

\section{Journals}

\section{Édition électronique}

URL : http://journals.openedition.org/variations/410

DOI : 10.4000/variations.410

ISSN : 1968-3960

\section{Éditeur}

Les amis de Variations

Référence électronique

Mikkel Bolt Rasmussen, «Remarques éparses (en style marxiste occidental) sur l'art contemporain, ses problèmes et ses contradictions », Variations [En ligne], 17 | 2012, mis en ligne le 15 octobre 2012, consulté le 20 avril 2019. URL : http://journals.openedition.org/variations/410 ; DOI : 10.4000/

variations. 410

Ce document a été généré automatiquement le 20 avril 2019.

Les ami•e•s de Variations 


\title{
Remarques éparses (en style marxiste occidental) sur l'art contemporain, ses problèmes et ses contradictions
}

\author{
Mikkel Bolt Rasmussen \\ Traduction : José Chatroussat
}

1 Ne serait-ce qu'un bref moment, examinons l'art contemporain à la lumière de l'idée marxiste occidentale, à savoir comme un instrument de résistance, comme ce que Herbert Marcuse appelait «le grand rejet » ou Guy Debord «l'art du changement » ${ }^{1}$. Examinons l'art contemporain dans cette optique, même si cela peut s'apparenter à casser des noix avec une masse ou peu s'en faut, et même si cela intervient éventuellement trop tard. Que voyons-nous? Son visage n'apparaît pas particulièrement avenant. Il semble que les formes traditionnelles d'opposition ne soient plus du tout disponibles. Les images visuelles aussi bien que les mots ou la musique semblent rater les anciens effets aliénants et sont rarement antagonistes avec l'ordre dominant. Où que nous jetions notre regard, c'est la complicité entre les institutions artistiques et le pouvoir établi qui est en général flagrante. Depuis 1989, l'économie spéculative du capitalisme néolibéral engloutit des sommes d'argent énormes dans le marché de l'art. La conséquence pour l'art aujourd'hui est qu'il se trouve étroitement lié à la circulation transnationale du capital. En même temps, les gouvernements nationaux, les régions et les villes utilisent l'art comme un instrument marchand dans la compétition fébrile pour avoir de la main d'œuvre, des investissements et des touristes. Ce développement de liens toujours plus étroits entre l'art et le capital, et entre l'art et l'ordre dominant, est incontestablement la tendance prédominante dès qu'on aborde l'art contemporain.

2 Mais en même temps, il est important de relever que l'espace de l'art se caractérise toujours par la présence de représentations variées du politique et par des tentatives pour utiliser le champ artistique comme un point de départ pour rendre visibles des conflits qui ont été marginalisés dans la sphère publique dominante. Par exemple, des 
expositions " politiques » se tiennent régulièrement et même de grandes institutions ont facilité ici ou là des expositions "politiques » et montré des représentations artistiques de thèmes "extérieurs à l'art ». Je peux mentionner rapidement la liste des plus importantes d'entre elles depuis 2004: «Les Interventionnistes» au MASS MoCA; «Communisme» au Projects Arts Centre de Dublin; "Populisme» au Museum for Contemporary Art d'Oslo et au Frankfurter Kunstverein parmi d'autres endroits ; «Die Regierung» ("Le Pouvoir») au MACBA et à la Sécession à Vienne parmi d'autres lieux; "Now-Time Venezuela» à l'U.C. Berkeley Art Museum; «Revolution is not a Garden Party » à la Trafo Gallery de Budapest et la Galerija Miroslav Krajevic à Zagreb entre autres ; « Forms of Resistance » au Van Abbe Museum d'Eindhoven ; «Signs of Change » à l'Exit Art de New York et « Asking We Walk : Voices of Resistance » au Den Frie Udstilling de Copenhague. Depuis la fin des années 1990, il y a donc eu un intérêt plus important pour l'art collectif et antagoniste, et pour les projets d'art activiste qui ont été invités dans les institutions artistiques et ont été l'objet d'expositions majeures.

Si nous considérons les biennales et les documenta comme repères significatifs dans la période qui a suivi 1989, nous pouvons alors observer un mouvement partant d'expositions très traditionnelles au début des années 1990, avec une prépondérance des peintures réalisées par des hommes blancs d'âge moyen, par exemple la documenta 9 de Jan Huet en 1992, et allant vers des expositions postcoloniales et critiquant la mondialisation à la fin des années 1990. Par exemple en 2002, la documenta 11 d'okwui Enwezor et autres, fut une tentative d'initier un renversement explicite de l'art historique colonial et de la hiérarchie politique, et un lieu où l'art contemporain, en interaction avec les courants critiques de la philosophie, de l'urbanisme et de l'économie, traçait les grandes lignes des défis et des perspectives postcoloniales². Bien sûr, nombre des expositions qui ont été présentées comme " politiques » ne l'ont été que de façon très limitée. La référence au « politique » et l'inclusion de projets activistes a été la plupart du temps un gadget promotionnel, une tentative pour «se conformer aux désirs des musées, des périodiques et du marché pour donner une représentation visuelle du politique », ou tout simplement une manœuvre pour mettre hors jeu tout geste potentiellement radical ${ }^{3}$. Malgré tout, il y a eu des tentatives pour attirer l'attention sur des questions politiques pressantes ou pour contester la manière dont nous les pensons.

Cependant, les expositions sont confrontées au problème qu'elles se tiennent en l'absence d'un contexte politique où les projets pourraient potentiellement avoir une signification au-delà de l'enclave de l'institution artistique. Paradoxalement, cela se présente comme si certains secteurs de l'institution artistique étaient gorgés de représentations des conflits et des luttes politiques parce que, dans une certaine mesure, elles n'apparaissent nulle part ailleurs. Il n'existe pas un grand domaine politique progressiste, global, avec une nouvelle façon de penser ou une articulation des résistances, une discussion des stratégies efficaces pour endiguer le nouveau régime dressé contre toute dissidence. Ce régime, qui se déploie de Copenhague à Gaza en passant par Athènes, est censé garantir aux riches de survivre aux crises et aux catastrophes à venir, qu'elles prennent la forme d'attaques terroristes, de vagues migratoires ou de désastres climatiques. En l'absence de projets politiques radicaux viables, il semble que ce soit aux marges de l'institution artistique que des contre-paradigmes et des politiques alternatives puissent rester vivantes, si ce n'est autrement que comme représentations des conflits. 


\section{Contradictions}

5 En d'autres termes, nous sommes confrontés à une situation pleine de contradictions. C'est bien sûr en partie ce que Benjamin, Marcuse, Lefebvre et d'autres ont relevé en leur temps, lorsqu'ils proposèrent leurs analyses critiques de l'art d'avant-garde et de son ambivalence problématique quant à l'autonomie de l'art dans la première moitié du vingtième siècle. Ils étaient tous conscients, de façon aiguë, que l'art moderne impliquait une promesse de bonheur, allant au-delà de la société capitaliste bourgeoise établie, tandis qu'en même temps il la consolidait. C'est ce que Marcuse résumait par sa formulation du caractère positif de l'art. D'un côté, l'autonomie de l'art fournit à l'artiste une liberté qui est à la fois la préfiguration d'une pratique libre dans une société libre et une critique de la société existante non libre. D'un autre côté, l'art moderne légitime toujours la société dans laquelle il existe. Comme l'écrit Marcuse, la sphère indépendante de l'art est positive puisqu'elle est « compatible avec le mauvais présent $»^{4}$. Cette dualité dialectique a été une plate-forme pour les avant-gardes historiques afin d'attaquer l'institution de l'art, de mener leurs tentatives paradoxales de dépassement de l'autonomie artistique et de réaliser la liberté de l'art en-dehors de sa base institutionnelle, comme une composante de la transformation radicale de la vie humaine. L'imagination anarchiste qui avait hiberné dans l'art mais disparu dans le reste de la vie humaine à cause de la réalisation de la vie moderne devait être débridée et mise à la disposition de tout le monde 5 .

6 Mais comme nous le savons tous trop bien, le projet ambitieux des avant-gardes a échoué. Tout comme le mouvement révolutionnaire concomitant des années 1920, il a fait l'expérience de ce que la contre-révolution a tout renversé et transformé dans une perspective où chaque chose redevient la même qu'avant. Les grandes tentatives de l'avant-garde de briser la frontière entre l'art et la vie ont fini par n'être que la négation immanente de l'autonomie de l'art et non la révolution désirée.

7 Rétrospectivement, c'est la capacité de l'institution à subsumer même les tentatives extrêmement radicales de rupture qui demeure lorsque nous portons notre regard sur l'art du vingtième siècle. L'anti-art des avant-gardes s'est intégré à la société bourgeoise capitaliste et l'a renforcée. Ce fut la conclusion des avant-gardes des années soixante, des situationnistes, et des théoriciens des avant-gardes des années soixante-dix, Peter Bürger et Manfredo Tafuri, les plus sagaces et par conséquent les plus résignés lorsqu'ils ont tourné leur regard vers les avant-gardes de l'entre-deux-guerres et leur destin ultérieur' ${ }^{6}$. Les avant-gardes n'ont pas eu de chance : le goût dominant s'est employé à récupérer ou à détourner tout ce à quoi il s'est trouvé confronté, en grand ou en petit. Rien n'était autorisé à exister seul, tout était censé faire partie du spectacle.

\section{L'industrie culturelle et le spectacle aujourd'hui}

Les contours de ce développement sous l'égide de la récupération de la théorie de l'avantgarde ci-dessus mentionnée nous sont familiers, mais aussi nombre de textes tout à fait centraux dans la partie du marxisme occidental concernant l'art tel que le chapitre d'Adorno et Horkheimer sur l'industrie culturelle dans Dialektik der Aufklärung ( $L a$ dialectique de la Raison) et tel que l'excoriation de Godard par les situationnistes dans « Le rôle de Godard ». Les deux penseurs allemands analysaient comment l'art autonome a été intégré dans l'industrie culturelle ${ }^{7}$. Selon Adorno et Horkheimer, la production capitaliste 
et la logique de l'administration ont subsumé l'art en production marchande et ont ainsi suspendu la résistance à l'aliénation et à la valeur d'échange qui était inhérente à l'art. La question exposée par Adorno et Horkheimer est soulevée par nous aujourd'hui, avec non moins de force, alors que l'art et la culture, encore bien plus que dans les années 1940, sont devenus des marchés importants avec une pléthore de niches et de styles différenciés sur une base marchande et alors que l'art contemporain fonctionne clairement comme le secteur Recherche et Développement pour les autres branches de l'industrie culturelle. Dans le cadre de l'offensive néolibérale qui a balayé le monde depuis la fin des années 1970, il y a eu une commercialisation croissante de l'institution de l'art. Même si l'art n'a pas dû répondre directement à une exigence de profitabilité, il a dû au minimum se défaire de ses dernières références à l'autonomie et non seulement coopérer avec le monde des affaires mais le prendre comme modèle et instrument d'efficacité et mettre en œuvre les notions d'efficacité et de quantification de l'industrie privée ${ }^{8}$. Entre autres façons, cela a pu être fait en s'assurant que les institutions de l'art attiraient les investissements pouvant contribuer au tourisme culturel et aux projets urbains d'embellissement qui ont fait intégralement partie des processus de gentrification, lesquels ont joué un rôle important dans la restructuration néolibérale de nombreuses grandes villes. Une telle activation ou animation de la ville, où l'art est un événement, était un rêve qui est devenu réalité pour les managers de la production du capitalisme néolibéral ${ }^{9}$.

Comme Julian Stallabrass l'écrit dans son Art Incorporated, le néolibéralisme a peut-être eu son impact le plus explicite dans les expositions gigantesques et les biennales qui peuvent être considérées de maintes façons comme des supermarchés, avec des œuvres d'art circulant librement, sans profondeur historique ni spécificité régionale: un art contemporain global, à l'instar d'une marque universelle qui effectue une homogénéisation, un évidage des différentes cultures et qui remplace l'histoire par la diversité géographique, comme si c'était une garantie de différence au lieu d'être un déguisement pour l'inévitable standardisation du marché ${ }^{10}$.

Dans leur analyse des films de Godard, Debord et les situationnistes avaient à la fois compris que l'art expérimental consolidait le spectacle - selon eux, les films de Godard représentaient une pseudo-critique formelle qui entravait une critique authentique de l'art et de la coupure de la vie par le spectacle en activités spécialisées - et ils montraient la façon dont la forme marchande sur le marché spectaculaire du capitalisme avait infiltré tous les aspects de la vie ${ }^{11}$. Ils définissaient ce spectacle comme étant l'ordre totalitaire anonyme de l'argent, un monde dépeuplé existant au-delà de l'influence humaine, le monde de la marchandise comme processus de représentation. La réalité, selon les situationnistes, a été transformée en images au-delà du contrôle humain et tout dialogue a été remplacé par le monologue de l'image. Même si cela est le résultat d'une pratique sociale complexe, l'image agit comme un être indépendant.

11 En accord avec l'interprétation marxiste fondamentale de la société capitaliste, les situationnistes pensaient que le besoin totalitaire du capitalisme de tout individualiser et de privatiser tout ce qui est collectif conduit à une société poreuse et incohérente qui ne se maintient que par un bombardement permanent de slogans, de publicités et de marques.

12 L'intensité avec laquelle nous sommes tous agressés par les images n'a pas diminué depuis la fin des années 1960 lorsque Debord et les autres situationnistes analysaient de façon critique la société du spectacle. Ce qui s'est produit, en revanche, c'est que les 
machines qui produisent des images fonctionnent de plus en plus vite pour recouvrir une socialité de plus en plus usée. Les preuves que la société s'effondre sont visibles partout. Les images alarmistes et les publicités sont faites pour cacher une texture sociale évidée et standardisée où l'individu isolé et commercialisé est esclave des offres stupides du système du divertissement, de la mode, de l'information et de la technologie.

En revenant à la critique de l'art faite par le marxisme occidental des dimensions plus étriquées de l'art institutionnel, nous devons reconnaître que le rejet par les situationnistes de la pseudo-critique de Godard est également toujours pertinente. Après tout, les expositions "politiques » mentionnées plus haut indiquent clairement l'écart entre la signification de ces expositions se voulant dans un certain sens critique des institutions ou du système, et leur fonction véritable de légitimation de ce qui existe. Comme l'a dit l'artiste australien Oliver Ressler au public lors d'une intervention en lien avec l'exposition «Asking We Walk» au Den Frie Udstilling, le président de Taïwan Ma Ying-Jeou a été par exemple très enthousiaste au sujet de la partie de la biennale de Taipei en 2008, "A World Where Many Worlds Fit » organisée par Oliver Ressler, sur le mouvement altermondialiste et ses manifestations anticapitalistes. Selon le président, chaque biennale qui se respecte se doit d'être « créative, pleine d'énergie, sensible et en particulier rebelle». Cette déclaration démontre évidemment la capacité de l'establishment à subsumer la critique et met en exergue l'existence de la "critique " comme une preuve de pluralisme et d'ouverture. Cette dimension positive est aussi pertinente aujourd'hui qu'elle l'était durant la Guerre froide quand les Etats-Unis mettaient en scène l'art moderne, en particulier sous la forme de l'expressionnisme abstrait, comme une expression de la liberté dans le monde capitaliste (contrairement au réalisme socialiste de propagande de l'Union soviétique). Comme l'a diagnostiqué Hal Foster dans "Against Pluralism», le pluralisme dans l'art contemporain doit être largement considéré comme une légitimation d'un relativisme débridé affirmant ostensiblement que tout est permis ; ce qui, en réalité, neutralise les approches critiques et n'est qu'une version actualisée de ce que Marcuse appelait « la tolérance répressive ${ }^{12}$.

\section{PRATIQUE DE L'(IM)POSSIBLE}

14 Essayons de changer de perspective en allant de l'art institutionnel structuré vers la pratique orientée pour porter un regard plus précis sur les approches variées qui ont été utilisées au cours des dix ou quinze dernières années dans le secteur critique le plus intéressant de l'art contemporain. Une des approches les plus intéressantes a été ce qu'on a appelé l'art relationnel décrit par Nicolas Bourriaud comme une tentative pour produire de nouvelles conditions sociales, comme réponse à la destruction des relations humaines que Bourriaud considère comme s'étant installées dans la société moderne tardive. Chez des artistes comme Rirkrit Tiravanija, Philippe Parreno et Carsten Höller, Bourriaud a découvert un intérêt intense pour «l'interaction, la coexistence et les réseaux relationnels", comme lorsque Tiravanija "organise un dîner au domicile d'un collectionneur d'art où il met à disposition toutes les choses nécessaires pour confectionner une soupe thaï $»^{13}$. Sous l'angle de l'histoire de l'art, l'esthétique relationnelle a été une voie pour aller au-delà de la critique de la représentation des années 1980 qui avait pris originellement la forme d'appropriations critiques et de déplacements des images des mass media. Mais à la fin des années 1980, cela s'est traduit par de brillants objets d'art impossibles à distinguer des marchandises de la société du 
spectacle, et par la manipulation de la sphère politique publique étroite. Après que l'art critique de la représentation des années 1980 a été préoccupé par les différentes théories post-modernes des signes, des textes et de la simulation, il y eut ensuite un tournant dans les années 1990 vers un mouvement performatif, à l'écart de la représentation et l'objet (d'art) vers les interactions sociales complexes et fluctuantes.

15 Mais, comme l'ont écrit Hal Foster et Stewart Martin parmi d'autres, l'esthétique relationnelle se caractérisait par une idée naïve de la présence et une confiance grandement exagérée en l'espace de l'art qui était supposé constituer une enclave socialement harmonieuse et non soumise au désordre du monde environnant ${ }^{14}$. Bourriaud en vint à considérer l'art contemporain comme un espace qui était " protégé à l'égard de l'uniformisation des comportements humains». De façon relativement rapide, l'esthétique relationnelle et donc ses types de relations non-accusatrices en vinrent à être perçues comme rien de plus que des Relations Publiques pour l'institution de l'art et le petit groupe des agents les plus privilégiés. La socialité vague et générale qui avait pris forme dans les œuvres relationnelles ne défiait en aucune manière l'institution qui n'eut aucune difficulté à réduire des relations en apparence si transitoires en objets d'art, marchandises et représentations de la socialité.

Une autre tendance plus intéressante dans l'art contemporain a été ce qu'on pourrait appeler l'art d'intervention-orienté-vers-une-solution qui entreprit de proposer des solutions à des problèmes politiques et sociaux compliqués et de créer de nouvelles formes collectives d'interaction sociale. Cet art partage l'aversion de l'art relationnel pour l'art critique de la représentation des années 1980. Depuis, dans une mesure encore plus grande que l'art relationnel, il est productif et propose résolument des modèles spécifiques de coopération et des manières de résoudre des problèmes sociaux particuliers. Des artistes comme Kenneth A. Balfelt, Superflex, Wochenklausur ou Asyl Dialog Tanken utilisent l'imagination artistique pour envisager, débattre et souvent même résoudre des problèmes de nature sociale ou économique - par exemple en donnant la possibilité à des toxicomanes de Copenhague de se piquer dans des conditions décentes, en remédiant au manque de refuge pour les prostituées à Zurich, en développant une plante pour le marché africain qui permet de transformer les déchets humaines ou animales en gaz, et la mise en place d'un centre culturel et d'un abri pour les immigrés et ceux qui cherchent un abri à Copenhague.

$17 \mathrm{Au}$ lieu d'une approche d'avant-garde qui problématise et s'oppose aux discours artistiques et politiques, nous avons là un échange pragmatique entre l'art et d'autres domaines. La relation antagonique de l'avant-garde avec les acteurs politiques et économiques dominants comme l'Etat et les entreprises multinationales est remplacée par une collaboration en vue de parvenir à des solutions à des problèmes concrets, des inégalités et des exclusions. D'une façon caractéristique de cette tendance, les Superflex décrivent leur art comme constitué de " tools " (" outils»); et Balfelt se réfère à l'idée de Charles Esche de «modestes propositions » où l'artiste, homme ou femme, à travers son approche particulière, en utilisant sa créativité et son imagination artistique, crée des alternatives, des propositions modestes pour résoudre des problèmes concrets ${ }^{15}$. Les projets sont ainsi résolument constructifs. L'objectif premier n'est pas de lancer des innovations formelles ou de dénoncer les procédés douteux de l'art institutionnel. Leurs projets n'ont rien à voir avec les transgressions avant-gardistes. Au contraire, ils visent à utiliser l'art (et le soutien public à l'art) comme plate-forme pour réaliser une œuvre constructive à l'extérieur de l'espace traditionnel de l'institution. Indiscutablement, ces 
projets ne sont pas seulement conscients mais aussi, de multiples façons, des efforts pertinents qui aident à donner une visibilité à des problèmes spécifiques négligés et qui utilisent l'autonomie de l'art pour aborder des problèmes extérieurs à l'art.

Mais, à la lumière de l'histoire du marxisme occidental concernant le potentiel transgressif de l'art moderne, ces projets risquent d'apparaitre plus pragmatiques et administratifs que véritablement critiques. Il y a largement matière à se compromettre avec le système établi en coopérant avec lui dans l'espoir de réparer certains dégâts qu'il a provoqués. Une véritable critique du système existe rarement. Les causes des problèmes ne sont pas exposées. Ainsi, le risque est à l'évidence que l'art-orienté-vers-des-solutions collabore avec ce qui existe déjà et, de cette manière, ne se situe que dans ce que Jacques Rancière appelle l'espace de la politique consensuelle où tout simplement « est ce qui est $»^{16}$. Le projet Guarana de Superflex en est un excellent exemple ${ }^{17}$. Un groupe d'agriculteurs brésiliens cultivant le guarana était sous la pression de grandes firmes multinationales faisant baisser le prix de leurs récoltes. En liaison avec eux, Superflex a lancé et développé une boisson non alcoolisée, Guarana Power, dont une plus grande part que d'habitude des bénéfices revient aux agriculteurs. Ce projet attachant est symptomatique de ce type d'art qui ne se positionne pas de façon critique vis-à-vis de la structure déjà en place, en l'occurrence la société capitaliste et son marché qui apparaît comme une prémisse non problématique pour le projet. La position de Superflex semble être que, en développant une marchandise se situant légèrement sur un terrain de gauche avec des connotations politiques modérées - «Je bois politiquement correct » - et en la vendant sur le marché, les agriculteurs vont réussir dans leur projet. Ils proposent de résister mais d'une manière moins rien que radicale. Au lieu de développer des modèles de production et d'échange vraiment alternatifs ou de critiquer la dépendance et la réification générées par le capitalisme, les Superflex interviennent sur le marché capitaliste comme des entrepreneurs actifs recrutant de nouveaux travailleurs.

Sous ce rapport, en considérant l'art contemporain au travers du prisme du marxisme occidental et des avant-gardes, une pratique d'intervention se situant davantage dans une logique de négation et de confrontation apparaît plus pertinente. Elle agit sous les noms de terrorisme sémiotique, de guérilla de communication ou de media tactiques ${ }^{18}$. Plus ou moins inspirée par les photomontages de Dada et le détournement des situationnistes, c'est une manière de s'approprier les images ou techniques existantes et de les utiliser dans d'autres buts. En principe, ce ne sont pas des activités qui ont nécessairement un lien direct avec l'institution de l'art mais différentes sortes d'activisme, par exemple les campagnes contre les ateliers de surexploitation, les luttes pour les droits des animaux et sur le changement climatique. Dans les années 2000 cependant, il y a eu une plus large exposition de cette approche au sein des institutions artistiques qui voulaient être associées à l'authenticité que représentait cette pratique ou cette tactique ${ }^{19}$. Comme le décrit Naomi Klein dans No Logo, l'idée sous-jacente derrière les media tactiques est que les grands conglomérats et grandes entreprises subiront le plus de dommages si leur image publique ou leur identité visuelle est sujette à une attaque ou à une manipulation ${ }^{20}$. Une telle attaque a été lancée en 2003 avec la Nikeplatz par 0100101110101101.org, à partir de leur page d'accueil et d'un container d'information rouge pimpant installé sur la Karlsplatz à Vienne. Il y était présenté le dernier projet de Nike dont l'idée était d'acheter la place, de la renommer Nikeplatz et d'y mettre une grande sculpture ayant la forme du logo iconique de la compagnie, le Swoosh. Le projet était bien sûr un commentaire sur la vague néolibérale à l'œuvre de gentrification et de privatisation de plus en plus d'espaces 
dans la ville passant du domaine public à la propriété privée. Par exemple les noms de stades sportifs publics ou municipaux sont vendus à des firmes et à des sponsors privés.

Un autre exemple de ce type d'activisme artistique est illustré par les Yes Men qui ont développé une pratique qu'ils appellent «Identity correction». Ils prétendent expressément être des représentants d'institutions publiques comme l'Organisation Mondiale du Commerce (OMC) et ils les critiquent en exagérant et en caricaturant leurs politiques. Ils obligent ainsi les firmes et les institutions à entretenir des rapports avec des initiatives problématiques ou de fâcheuses affaires en cours. En 2002 les Yes Men se sont présentés par exemple comme des représentants de l'OMC à une conférence de comptables en Australie où ils ont annoncé que l'organisation allait se transformer en l'Organisation de Régulation du Commerce dont le but était d'aider les peuples au lieu du capital. Par le truchement de telles fausses déclarations, l'espoir est de pouvoir troubler momentanément ou défier le «naturel » dont s'est doté le capitalisme néolibéral depuis 1989. Et si cela réussit, il peut y avoir une sorte de désidentification avec les logos omniprésents représentant aussi bien les grandes compagnies que les politiciens. Il y a là même peut-être une ouverture de telle sorte qu'il devienne clair que les choses peuvent apparaître différentes.

Cependant, la question est de savoir si cet activisme, dans son utilisation du spectaculaire, finira par conforter la représentation actuelle sous-jacente du système et son fonctionnement, comme un désaccord interne au système, qui montre seulement que la société laisse de la place à la critique et aux idées bizarres et cinglées. Un genre de Michael Moore de l'art contemporain. Comme l'écrivain et artiste norvégien Matias Faldbakken l'a montré d'une façon divertissante et impitoyable dans ses romans, The Cocka Hola Company et Macht und Rebel, l'approche tactique des media est souvent dangereusement proche d'apporter une renommée supplémentaire et du coup, font de la publicité aux compagnies dont l'image a été déformée et qui sont en fait intéressées d'être associées à une aura de "mordant", de créativité et même d'illégalité ${ }^{21}$. La question est de savoir si la marque Nike a souffert d'un quelconque préjudice ou si elle est tout simplement encore plus fortement ancrée dans les cerveaux engourdis et blasés des consommateurs lorsque 0100101110101101.org a lancé Nikeplatz et modifié l'image de Nike pendant un bref moment.

22 Au-delà des différentes frontières significatives entre art relationnel, art orienté vers une solution et l'art d'intervention négative, il est clair que les pratiques mentionnées se caractérisent par une aversion partagée à l'égard des stratégies d'ensemble et des programmes globaux pour mettre de préférence l'accent sur la petite échelle, le temporaire et le tactique. Indiscutablement, il y a des raisons pour examiner de façon critique les projets beaucoup trop vastes et radicaux des avant-gardes qui escomptaient toutes parvenir vraiment à de grands résultats. Mais la question se pose encore de savoir si quelque chose est resté au sein de l'art contemporain de cette résistance que l'art était supposé incarner. Ces pratiques semblent pâtir du révisionnisme anticommuniste général accompagné de la peur de la révolution qui a fleuri de façon plus ou moins incontestée après l'écroulement de la dictature du parti de l'Etat capitaliste en Union soviétique et qui assimile les projets politiques et artistiques alternatifs au totalitarisme au profit d'une acceptation aveugle de la misère actuelle ou pour des remaniements très limités dans ce domaine ${ }^{22}$. L'art contemporain des années 1990 et 2000 n'a pas contesté l'horizon qui voyait le jour se lever avec les soulèvements postmodernes des années 1980 et fut confirmé par la chute du Mur en 1989 lorsque la lutte idéologique de la Guerre froide fut 
remplacée par un scénario post-idéologique caractérisé par la méfiance à l'égard des grands récits d'émancipation. Rétrospectivement, il est clair que cette liquidation des discours politiques s'est installée comme une tentative de grande envergure pour donner un statut hégémonique au néolibéralisme. Les (autres) grandes idéologies politiques ont été mises à la porte dans l'optique de naturaliser la phase néolibérale du capitalisme. Et ce projet a réussi dans une grande mesure. De 1989 à 1999, le néolibéralisme, dans l'ensemble, n'a pas été contesté ; toute référence à des alternatives ayant des tendances communistes a été écartée comme conduisant au totalitarisme. L'ouverture que les grandes protestations lors des sommets de Seattle, Prague, Gênes et d'autres endroits semblaient rendre possible, au tournant du siècle, a été refermée avec une grande force par l'ordre établi au travers de l'installation d'un régime anti-rébellion mis en scène dramatiquement après le 11 septembre comme la guerre contre le terrorisme; et il est aujourd'hui légitimé en référence au terrorisme, à l'immigration et aux changements climatiques. Rétrospectivement, il est clair que l'esthétique relationnelle et l'art d'intervention ont souscrit dans diverses mesures à ce discours post-idéologique, avec leurs idées de micro-utopies, de propositions spécifiques pour apporter des solutions et de tactiques éphémères qui ne nourrissaient aucune vision large pour un futur différent. Les antagonismes des années passées furent supplantés par des efforts limités pour établir des zones temporaires de libre interaction dans l'espace de l'art ou pour perturber en douceur la circulation des représentations et des marchandises. La critique générale de l'état actuel des affaires apparaît comme une chose appartenant au passé.

Mais peut-être qu'il est simplement et complètement non-contemporain de considérer l'art contemporain à la lumière de la partie du marxisme occidental en rapport avec l'art? Les envois de rapports grandiloquents aux morts peuvent bien sûr être rejetés comme désespérément rattrapés par des modes de penser modernes et essentialistes gênants qui sont totalement incapables d'appréhender le présent. Il est tout à fait concevable que les choses en soient ainsi. Pourquoi insister perversement sur les impossibilités et se focaliser sur le mauvais côté des contradictions en se référant aux grandes défaites et aux illusions détruites du passé? N’avons-nous pas évolué? Et n'avons-nous pas laissé derrière nous ces orientations schématiques et chimériques pour acquérir une approche plus réaliste des perspectives que nous pourrions prendre aujourd'hui comme point de départ? À une époque où les événements révolutionnaires sont diffusés d'abord et avant tout sous la forme de signes vides de l'iconographie culturelle pop, dans laquelle Baader-Meinhof, Marx et Mao fusionnent dans une mixture exécrable - ce qui aboutit à saboter plutôt qu'à faciliter la mémoire, c'est précisément, de façon paradoxale, en insistant sur le potentiel de la répétition et des déplacements que nous pouvons œuvrer à prendre un nouveau départ, que nous pouvons créer une puissante nostalgie au sens de Walter Benjamin ${ }^{23}$.

Peut-être qu'il n'est pas si mauvais, après tout, d'être en désaccord avec le Zeitgeist, l'esprit du temps, peut-être que c'est précisément là que réside un potentiel - dans l'anachronique? Non pas que nous devrions aujourd'hui jouer le rôle de Marx en 1848, de Leviné en 1919 ou de Debord en 1968. Le vrai bouleversement est sans doute quelque chose qui vient toujours de l'extérieur et apparaît incompréhensible à l'ordre établi. 


\section{NOTES}

1. Le marxisme occidental a été le marxisme critique qui a surgi en Europe occidentale après 1917, en opposition et comme critique du communisme soviétique et de la dictature du parti à la tête du capitalisme d'Etat qui devint rapidement une réalité en Union soviétique. Les marxistes occidentaux tentèrent de formuler des alternatives au communisme soviétique qui se consolidait rapidement. Ils considérèrent avec scepticisme la conception de Lénine du parti et mirent en question l'idée que la Révolution russe soit un modèle applicable aux autres nations européennes. Dans cette tâche figuraient des figures majeures comme Georg Lukacs et Karl Korsch. Après la Deuxième guerre mondiale, l'application d'une critique de la société bourgeoise de consommation devint un des points centraux pour la génération suivante de cette tradition qui comptait Theodor W. Adorno, Henri Lefebvre et Cornelius Castoriadis. L'idée de l'art comme instrument de résistance a joué un rôle important pour nombre de marxistes occidentaux, par exemple plusieurs représentants de l'Ecole de Francfort, les philosophes français de «la vie quotidienne » et l'Internationale situationniste. Pour des présentations de cette tradition, voir Perry Anderson, Considerations on Western Marxism (London, NLB, 1971), Richard Gombin, Les origines du gauchisme (Pars, Seuil, 1971) et Russell Jacoby, Dialectics of Defeat: Contours of Western Marxism (Cambridge \& New, Cambridge University Press, 1981).

2. Dans Hegemonie im Kunstfeld. Die documenta-Austellungen d10, d11, d12 und die Politik der Biennalisierung (Cologne, Neuer Berliner Kunstverein \& Walter König, 2008), Oliver Marchart a recourt à une analyse du discours inspirée par Laclau et Mouffe pour analyser la documenta 10 et la documenta 11, comme tentative de donner à la sphère publique de l'art une fonction politique. 3. Brian Holmes, «Liar's Poker. Representation of Politics/Politics of Representation », Springerin, $\mathrm{n}^{\circ}$ 1, 2003, www.springerin.at/dyn/heft_text.php?textid=1276\&lang=en

4. Herbert Marcuse, Über den affirmativen Charakter der Kultur [1937], Kultur und Gesellschaft (Frankfurt, Suhrkamp, 1968), p. 86 ; trad.fr. Culture et Société, Paris, éd de Minuit, 1970.

5. C'est Jochen Schulte-Sasse qui, dans le texte «Imagination and Modernity : or the Taming of the Human Mind» (Cultural Critique, n5, pp. 23-48), décrit le projet de l'avant-garde comme une tentative pour libérer l'imagination anarchiste qui avait été domestiquée ou apprivoisée comme composante du processus de différenciation de la modernité.

6. CF Peter Bürger, Theorie der Avantgarde (Frankfurt, Suhrkamp, 1974) et Manfredo Tafuri, Progretto e utopia. Architettura e sviluppo capitalistico (Bari, Laterza, 1973).

7. Theodor W. Adorno et Max Horkheimer, «Kulturindustrie. Aufklärung als Massenbetrug », Dialektik der Aufklärung. Philosophische Fragmente [1944] ; trad. fr. La dialectique de la Raison, Paris, 1984, Max Horkheimer, Gesammelte Schriften. Band 5 (Frankfurt, Fischer, 1987), pp. 144-196.

8. CF Mark Rectanus, Culture Incorporated: Museums, Artists and Corporate Sponsorship (Minneapolis \& London : University of Minnesota Press, 2002), Chin Tao-Wu, Privatising Culture: Corporate Art Intervention since the 1980s (London \& New York, Verso, 2001) and Anthony Davies, « Take Me I'm Yours : Neoliberalising the Cultural Institution », Mute, vol 2, n5, 2007, pp. 100-113.

9. Pour des analyses de cette utilisation de l'art et des institutions artistiques, voir Lorenzo Vicario \& Pedro Manuel Martinez Monje, «The Guggenheim Effect », Shrinking Cities. Volume 2: Interventions (Ostfildern: Hatje Cantz, 2006), pp. 744-752 et Sharon Zukin: «How to Create a Culture Capital : Reflections on Urban Markets and Places ", Iwona Blazwick (éd) : Century City: Art and Culture in the Modern Metropolis (London : Tate Gallery Publishing, 2001), pp. 258-264. La reprogrammation des artistes comme avant-garde de la gentrification a été formulée de façon 
exemplaire par Richard Florida: The Rise of the Creative Class. And How It's Transforming Work, Leisure and Everyday Life (New York, Basic Books, 2002).

10. Julian Stallabrass : Art Incorporated: The Story of Contemporary Art (New York, Basic Books, 2002).

11. Internationale situationniste : «Le rôle de Godard », Internationale situationniste, $\mathrm{n}^{\circ}$ 10, 1966, pp. 58-59.

12. Hal Foster, «Against Pluralism », Recodings : Art, Spectacle, Cultural Politics (Seattle, Bay Press, 1985), pp. 13-32.

13. Nicolas Bourriaud, Esthétique relationnelle (Dijon-Quetigny, Les presses du réel, 1998).

14. CF Hal Foster, «Arty Party», London Review of Books, décembre 2003, pp. 21-22 et Stewart Martin, «Critique of Relational Aesthetics », Third Text, 2007, n 87, pp. 369-386.

15. Superflex \& Barbara Steiner (eds.): Tools (Cologne, Walter König, 2003) et Charles Esche, Modest Proposals (Istanbul, Baglam Publishing, 2005).

16. Jacques Rancière, Chroniques des temps consensuels (Paris, Seuil, 2005).

17. Pour une présentation de ce projet, voir Will Bradley, "Guarana Power ", Superflex et al. (eds), Self-organisation/counter-economic strategies (New York \& Berlin, Sternberg Press, 2006), pp. 311-333.

18. Certains des textes "classiques» de cette tactique comprennent le groupe autonome a.f.r.i.k.a, Luther Blissett \& Sonja Brünzels (eds), Handbuch des Kommunikationsguerilla (Hamburg, Verlag Libertäre Assoziation \& Schwartze Risse/Rote Strasse, 1997), Critical Art Ensemble, Digital Resistance : Explorations in Tactical Media (New York, Autonomedia, 2001) et Geert Lovink \& David Garcia, «ABC of Tactical Media », 1997, www.nettime.org/Lists-Archives/nettime-1-9705/ msg00096.html

19. CF la question thématique du Third Text $\left(n^{\circ} 94,2008\right)$ ayant pour titre «Whiter Tactical Media? » [ Faire dépérir les media tactiques » ?], édité par Gene Ray et Blake Stimson où ils partent de l'absorption graduelle des tactiques dans l'institution de l'art.

20. Naomi Klein, No Logo: Taking Aim at the Brand Bullies, (New York, Picador, 2000); trad. fr. No Logo, la tyrannie des marques, Paris, Actes Sud, 2001.

21. Matias Faldbakken, The Cocka Hola Company (Oslo, Cappelen Damm, 2001) et Macht und Rebel. Skandinavisk misantropi (Oslo, Cappelen Damm, 2002).

22. CF Domenico Losurdo, Il revisionismo storico. Problemi e miti (Bari, Laterza, 1996), Slavoj Zizek, Did Somebody Say Totalitarianism? Five Interventions in the (Mis)Use of a Notion (London \& New York, Verso, 2001).

23. Cf. Walter Benjamin: Das Passagen-Werk, (Frankfurt: Suhrkamp, 1974), pp. 39-41; trad.fr. Paris, capitale du XIXe siècle : Le livre des passages, Paris, Le Cerf, 1989.

\section{INDEX}

Mots-clés : critique du travail, correspondance, artistes, socialisme vert, travailleurs culturels, Wertkritik, Gorz André, dialogue, Vincent Jean-Marie, écologie, modèles alternatifs, Marx Karl, penser le Capital, ethos du travail, Eglise Orthodoxe, salariat, domination 


\section{AUTEURS}

\section{MIKKEL BOLT RASMUSSEN}

Professeur en art et cultural studies, Université de Copenhague. 